06

\title{
Характеристики дефектной моды одномерного СВЧ волноводного фотонного кристалла с металлическим включением в элементе, нарушающем его периодичность
}

\author{
(С) Д.А. Усанов, А.В. Скрипаль, А.А. Романов \\ Саратовский национальный исследовательский государственный университет им. Н.Г. Чернышевского, \\ 410012 Россия, Саратов \\ e-mail: usanovda@info.sgu.ru
}

(Поступило в Редакцию 18 мая 2016 г.)

Исследованы амплитудно-частотные характеристики коэффициента пропускания СВЧ волноводного фотонного кристалла с нарушением периодичности при наличии плоского проводящего включения малых размеров, частично заполняющего поперечное сечение волновода, внутри нарушенного слоя в зависимости от его местоположения. Показано, что введение проводящего включения малых размеров внутрь нарушенного слоя фотонного кристалла приводит к частотному сдвигу дефектной моды в запрещенной зоне в сторону низких частот, при этом максимальный сдвиг наблюдается при расположении металлического включения на границе или в центре нарушения в зависимости от толщины нарушенного слоя.

DOI: 10.21883/JTF.2017.06.44511.1898

\section{Введение}

Новые возможности при создании СВЧ устройств открываются при использовании периодических структур, получивших название фотонных кристаллов [1-3]. Эти структуры состоят из периодически расположенных элементов, размеры которых сравнимы с длиной волны распространяющегося в них электромагнитного излучения. В спектре такой структуры имеется частотная область, запрещенная для распространения электромагнитной волны - аналог запрещенной зоны в кристаллах. Введение нарушения в периодичности слоистой структуры приводит к появлению в запрещенной зоне фотонного кристалла резонансных частотных особенностей узких „окон“ прозрачности $[4,5]$. В этом случае говорят о существовании дефектной моды.

Возможности практического применения СВЧ фотонных кристаллов рассматривались в $[1,6-10]$. В качестве примеров приводятся высоконаправленные резонансные антенны, резонансные полости для размещения полупроводниковых детекторов, различные типы СВЧ фильтров, в том числе с управляемыми характеристиками.

Описанные в работах [11-13] методы измерения параметров полупроводниковых структур с использованием СВЧ фотонных кристаллов предполагали, что измеряемая структура полностью заполняет поперечное сечение волновода. Поскольку в этом случае измеряемая структура вызывает достаточно резкое изменение амплитудно-частотных характеристик (АЧХ) фотонного кристалла, обеспечивается высокая разрешающая способность и чувствительность к изменению параметров измеряемых структур. Однако локальность измерений при этом ограничивается площадью поперечного сечения волновода и для повышения локальности измерений требуется использовать волноводы более высокочастот- ного диапазона, что вызывает, как правило, определенные технические трудности.

В случае, когда вносимая в фотонный кристалл исследуемая структура лишь частично заполняет поперечное сечение волновода, для усиления отклика АЧХ фотонного кристалла на ее параметры, представляет интерес изучение влияния ее размеров и местоположения внутри предварительно созданной неоднородности, выполняющей роль микрорезонатора в фотонном кристалле [14]. Авторами [15] было показано, что создание большого числа малых воздушных включений внутри нарушенного слоя приводит к изменению АЧХ коэффициента пропускания фотонного кристалла, выражающемуся, в частности, на сдвиг пика пропускания в запрещенной зоне в сторону высоких частот, т.е. изменению частоты дефектной моды. При этом оказывается возможным описание АЧХ фотонного кристалла с использованием модели „эффективной“ среды. В качестве альтернативы воздушным включениям для регулирования АЧХ фотонного кристалла можно рассматривать введение в нарушенный слой металлических включений и связанные с этим изменения характеристик дефектной моды.

Целью настоящей работы являлось исследование влияния на АЧХ коэффициента пропускания СВЧ фотонного кристалла с нарушением периодичности путем введения внутрь нарушенного слоя плоского проводящего включения малых размеров, частично заполняющего поперечное сечение волновода.

\section{Модель волноводного фотонного кристалла}

Исследовался волноводный фотонный кристалл, состоящий из одиннадцати слоев, в диапазоне частот 


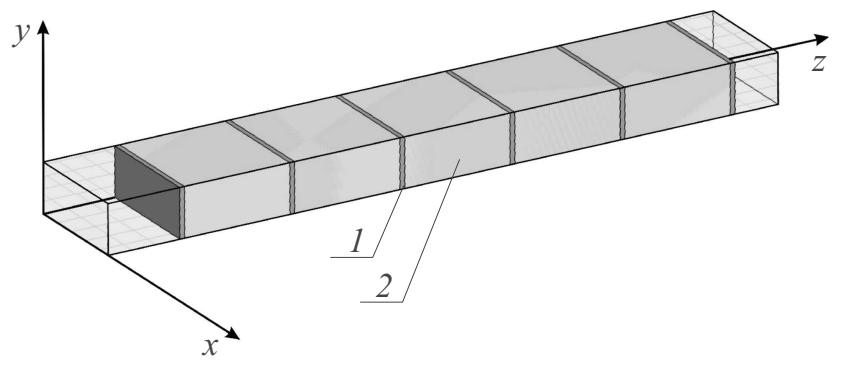

Рис. 1. 3D-модель волноводного фотонного кристалла без нарушения периодичности: 1 - керамика $\left(\mathrm{Al}_{2} \mathrm{O}_{3}\right), 2-$ фторопласт.
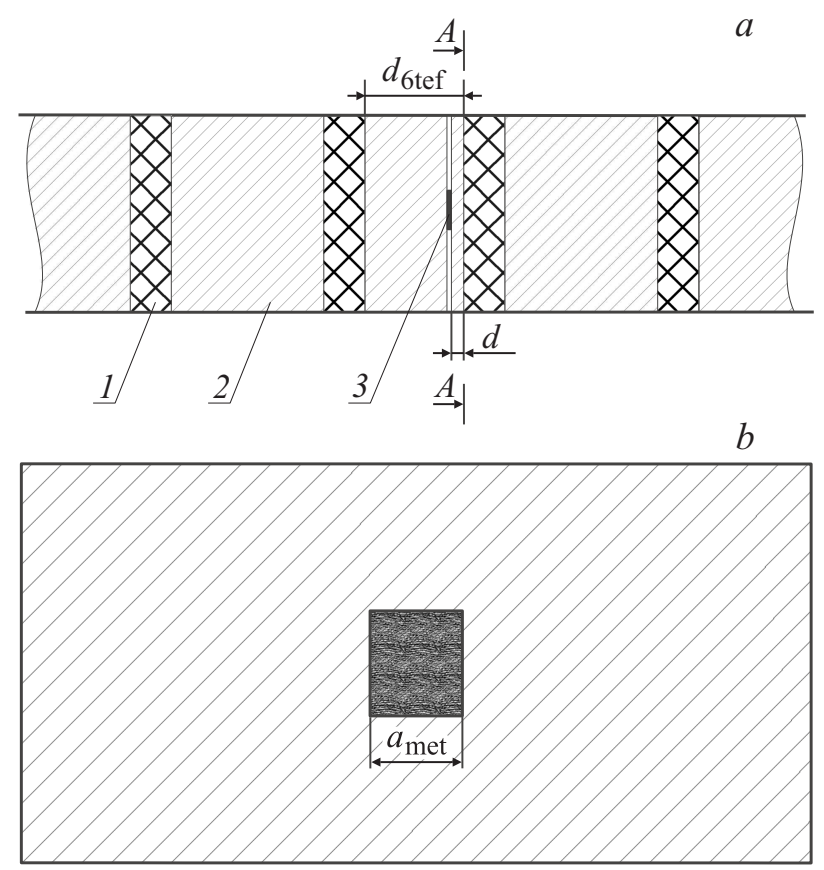

Рис. 2. Положение металлического включения в виде алюминиевой пленки в волноводе внутри нарушенного слоя: $a$ - продольное сечение волновода, $b-$ поперечное сечение волновода. 1 - слой поликора толщиной $1 \mathrm{~mm}, 2-$ слой фторопласта толщиной $22 \mathrm{~mm}, 3$ - металлическое включение, находящееся в нарушенном слое, удаленное от слоя поликора на расстоянии $d$.

8-12 GHz (рис. 1). Нечетные слои были выполнены из керамики $\left(\mathrm{Al}_{2} \mathrm{O}_{3}, \varepsilon=9.6\right)$, четные - из фторопласта $(\varepsilon=2.0)$. Толщина нечетных слоев $d_{\mathrm{Al}_{2} \mathrm{O}_{3}}=1 \mathrm{~mm}$, четных $d_{\text {tef }}=22 \mathrm{~mm}$. Слои полностью заполняли поперечное сечение волновода.

Размеры слоев были подобраны таким образом, чтобы запрещенная зона фотонного кристалла находилась в середине исследуемого диапазона частот 8-12 GHz.

Нарушение периодичности фотонной структуры создавалось изменением толщины $d_{6 \mathrm{tef}}$ центрального (шестого) слоя фторопласта, его толщина составляла 2.3 и $14.5 \mathrm{~mm}$.

Внутри нарушенного слоя в центре сечения волновода размещалось металлическое включение в виде алю- миниевой пленки толщиной $100 \mu \mathrm{m}$, имеющей форму квадрата со стороной $a_{\text {met}}$, равной $3 \mathrm{~mm}$ (рис. 2).

\section{Результаты компьютерного моделирования}

На основе численного моделирования с использованием программного обеспечения для трехмерного моделирования электромагнитных полей методом конечных элементов ANSYS HFSS исследовались АЧХ коэффициента пропускания фотонного кристалла с нарушением периодичности при наличии металлического включения, внесенного внутрь нарушенного слоя.

Как отмечалось выше, наличие нарушения периодичности фотонного кристалла приводит к возникновению дефектной моды на АЧХ коэффициента пропускания.

Для исследования влияния местоположения металлического включения в виде алюминиевой пленки внутри нарушенного слоя, выполняющего роль микрорезонатора в фотонном кристалле, на характеристики дефектной моды $[6,10]$ или „окна прозрачности“ [7] был выполнен расчет распределения напряженности электрического поля электромагнитной волны на частоте дефектной моды.

Результаты расчета распределения напряженности поля на частоте дефектной моды представлены на рис. 3.
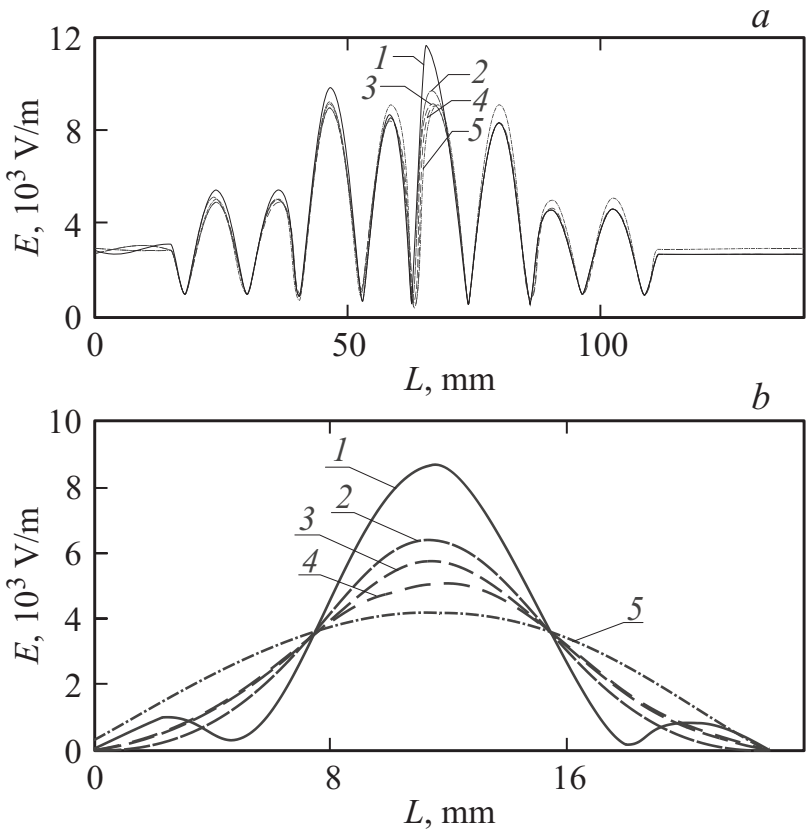

Рис. 3. Распределение напряженности электрического поля электромагнитной волны внутри фотонного кристалла для различного положения металлического включения $d$ внутри неоднородности и без включения: $a-$ вдоль направления распространения электромагнитной волны; $b-$ в поперечной плоскости волновода $(A-A)$ на рис. $2, f_{\text {res }}=10.095 \mathrm{GHz}$. Толщина нарушенного слоя $d_{6 \mathrm{tef}}=2.3 \mathrm{~mm} ; d, \mu \mathrm{m}: 1-0,2-$ $100,3-200,4-300,5-$ структура без металлического включения. 


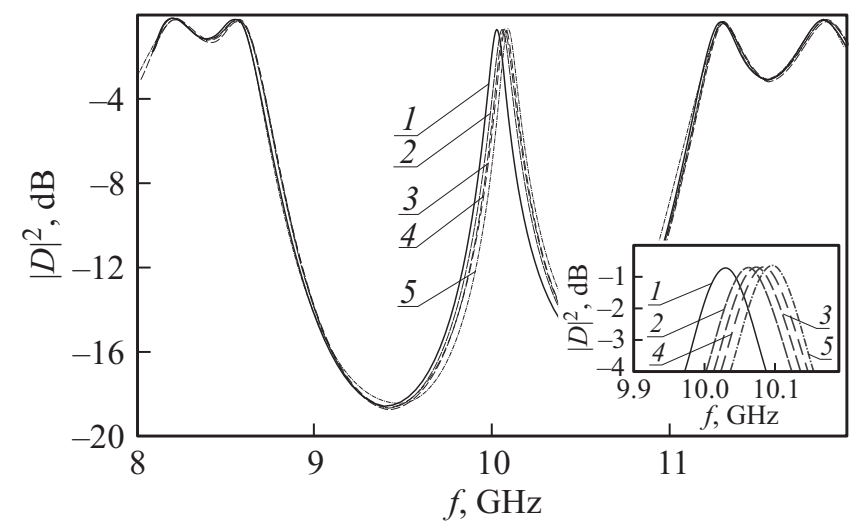

Рис. 4. АЧХ фотонного кристалла при различных положениях $d$ внутри нарушения металлического включения с размером $a_{\mathrm{met}}=3 \mathrm{~mm} ; d, \mu \mathrm{m}: 1-0,2-100,3-200,4-300,5-$ структура без металлического включения.

Результаты расчета напряженности электрического поля электромагнитной волны показывают чередование узлов и пучностей внутри фотонного кристалла вдоль направления ее распространения. При этом для выбранных параметров фотонного кристалла без металлического включения на частоте дефектной моды $f_{\text {res }}$ в центре нарушенного слоя наблюдается узел (кривая 5 на рис. 3 ).

Как следует из представленных на рис. 3 зависимостей, внесение металлического включения в виде алюминиевой пленки внутрь нарушенного слоя фотонного кристалла приводит к искажению в распределении напряженности электрического поля, при этом максимальное возмущение наблюдается при расположении металлического включения на границе нарушенного слоя.

Такое изменение распределения напряженности электрического поля может приводить к изменению положения резонансной особенности в запрещенной зоне фотонного кристалла.

Результаты расчета АЧХ фотонного кристалла при выбранных положениях металлического включения с размером $a_{\text {met }}=3 \mathrm{~mm}$ внутри нарушения представлены на рис. 4. На вставке рис. 4 представлены АЧХ фотонного кристалла вблизи частоты дефектной моды в запрещенной зоне фотонного кристалла.

Анализ представленных на рисунке зависимостей показывает, что наличие металлического включения в неоднородности приводит к сдвигу частоты дефектной моды $f_{\text {res }}$ фотонного кристалла в область низких частот относительно частоты дефектной моды $f_{0}$ при отсутствии металлического включения в неоднородности. При этом величина сдвига $\Delta f=f_{\text {res }}-f_{0}$ максимальна $(\Delta f=63 \mathrm{MHz})$ при расположении включения на границе нарушенного слоя $d=0$ и уменьшается при смещении металлического включения к центру неоднородности $(\Delta f=13 \mathrm{MHz}$ при $d=300 \mu \mathrm{m}), \Delta f$ минимально при расположении металлического включения в центре неоднородности $d=1.15 \mathrm{~mm}$.
Расчеты показывают, что созданная дефектная мода при толщине $d_{6 \mathrm{tef}}=2.3 \mathrm{~mm}\left(d_{6 \mathrm{tef}}<\lambda_{\text {res }} / 2\right.$, где $\lambda_{\text {res }}-$ длина волны на частоте дефектной моды) может быть реализована и при большей толщине $d_{6 \mathrm{tef}}=14.5 \mathrm{~mm}$ ( $\left.\lambda_{\text {res }} / 2<d_{6 \text { tef }}<\lambda_{\text {res }}\right)$, однако в этом случае происходит существенное изменение распределения напряженности электрического поля электромагнитной волны внутри фотонного кристалла вдоль направления ее распространения на частоте дефектной моды, приводящее к возникновению пучности напряженности электрического поля в центре нарушенного слоя.

Анализ АЧХ фотонного кристалла с толщиной нарушенного слоя $d_{6 \mathrm{tef}}=14.5 \mathrm{~mm}$ показывает, что наличие металлического включения в неоднородности и в этом случае приводит к сдвигу частоты дефектной моды фотонного кристалла в область низких частот. При этом величина сдвига максимальна при расположении включения в центре нарушенного слоя и минимальна при расположении металлического включения на границе неоднородности.

\section{Эксперимент}

Экспериментально исследовался фотонный кристалл, созданный в соответствии с описанной выше моделью и представляющий собой чередующиеся слои керамики и фторопласта. Измерение АЧХ коэффициента пропускания исследуемого фотонного кристалла в трехсантиметровом диапазоне длин волн проводилось с помощью векторного анализатора цепей Agilent PNA-L Network Analyzer N5230A.

Экспериментальные АЧХ фотонного кристалла при различных положениях металлического включения с фиксированным размером $a_{\text {met }}=3 \mathrm{~mm}$ внутри нарушения размером $2.3 \mathrm{~mm}$ представлены на рис. 5. На вставке рис. 5 представлены АЧХ фотонного кристалла вблизи частоты дефектной моды фотонного кристалла.

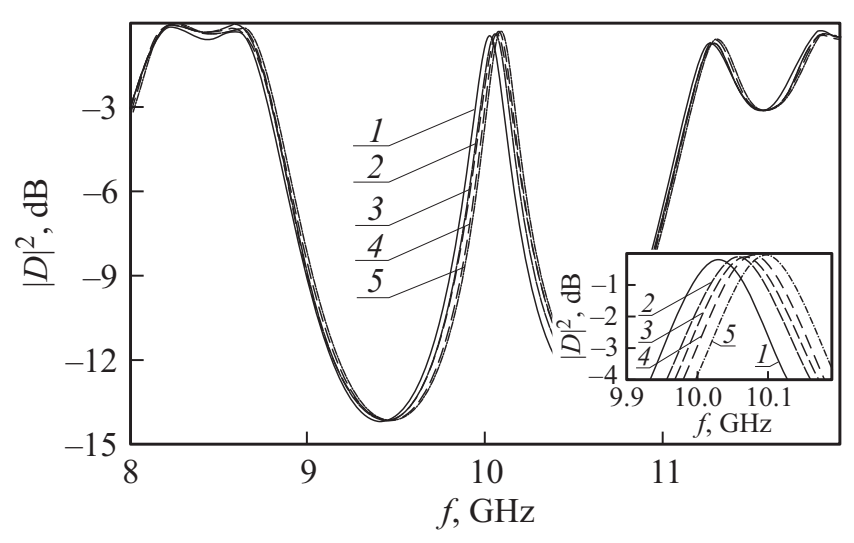

Рис. 5. Экспериментальные АЧХ фотонного кристалла при различных положениях $d$ внутри нарушения металлического включения с размером $a_{\mathrm{met}}=3 \mathrm{~mm} ; d, \mu \mathrm{m}: 1-0,2-$ $100,3-200,4-300,5-$ структура без металлического включения. 
Сравнение расчетных и экспериментальных АЧХ (рис. 4 и 5) фотонного кристалла при различных положениях металлического включения внутри нарушения при $d_{6 \mathrm{tef}}=2.3 \mathrm{~mm}$ свидетельствует об их хорошем количественном совпадении и подтверждает, что величина сдвига минимальна при расположении включения в центре нарушенного слоя и максимальна при расположении металлического включения на границе неоднородности.

Были измерены АЧХ фотонного кристалла с увеличенной толщиной центрального нарушенного слоя, равной $14.5 \mathrm{~mm}$, анализ которых подтвердил результаты компьютерного моделирования, свидетельствующие о том, что величина сдвига дефектной моды в этом случае минимальна при расположении металлического включения на границе неоднородности и максимальна при расположении металлического включения в центре нарушенного слоя.

\section{Заключение}

Таким образом, проведено численное моделирование и экспериментальное исследование влияния на АЧХ коэффициента пропускания СВЧ фотонного кристалла с нарушением периодичности введения плоского проводящего включения малых размеров внутрь нарушенного слоя.

Установлено, что введение плоского проводящего включения малых размеров внутрь нарушенного слоя фотонного кристалла приводит к сдвигу дефектной моды в запрещенной зоне в сторону более низких частот, при этом максимальный сдвиг наблюдается при расположении металлического включения на границе нарушения при толщине нарушенного слоя $d_{6 \mathrm{tef}}<\lambda_{\mathrm{res}} / 2$ и в центре нарушения при $\lambda_{\text {res }} / 2<d_{6 \text { tef }}<\lambda_{\text {res. }}$. Полученные результаты могут быть использованы, в частности, при конструировании СВЧ фотонных кристаллов с электрически управляемыми характеристиками.

Работа выполнена при финансовой поддержке Министерства образования и науки РФ (государственное задание № 1376 и 1575).

\section{Список литературы}

[1] Ozbay E., Temelkuran B., Bayindir M. // Progr. Electromagneti. Res. 2003. Vol. 41. P. 185-209.

[2] Gomez A., Vegas A., Solano M.A., Lakhtakia A. // Electromagnetics. 2005. Vol. 25. N 5. P. 437-460.

[3] Гуляев Ю.В., Никитов С.А. // Радиотехника. 2003. № 8. C. $26-30$.

[4] Yablonovitch E., Gimitter T.J., Meade R.D. // Phys. Rev. Lett. 1991. Vol. 67. N 24. P. 3380-3383.

[5] Usanov D., Skripal A., Abramov A., Bogolubov A., Skvortsov V., Merdanov M. // Proc. of 37th European Microwave Conf. Munich, Germany. 8-12th October 2007. P. 198-201.
[6] Gerard W. Burns, Thayne I.G., Arnold J.M. // Proc. of European Conf. on Wireless Technology, Amsterdam, Netherlands, 11-12th October 2004. P. 229-232.

[7] Hsien-Shun Wu, Ching-Kuang C. Tzuang // Proc. of 34rd European Microwave Conf. (Amsterdam, Netherlands, 12-14-th October 2004). 2004. Vol. 2. P. 1189-1192.

[8] Беляев Б.А., Волошин А.С., Шабанов В.Ф. // ДАН. 2005. T. 403. № 3. C. 319-324.

[9] Вендик И.Б., Вендик О.Г. // ЖТФ. 2013. Т. 83. Вып. 1. С. 3 28.

[10] Усанов Д.А., Мерданов М.К., Скрипаль А.В., Пономарев Д.В. // Известия Сар. гос. ун-та. Новая серия. Сер. 1. Физика. 2015. Т. 15. Вып. 1. С. 57-73.

[11] Усанов Д.А., Скрипаль А.В., Абрамов А.В., Боголюбов А.С., Скворцов В.С., Мерданов М.К. // Известия вузов. Электроника. 2007. № 6. С. 25-32.

[12] Гуляев Ю.В., Никитов С.А., Усанов Д.А., Скрипаль А.В., Постельга А.Э., Пономарев Д.В. // ДАН 2012. Т. 443. № 5. C. 564-566.

[13] Usanov D.A., Skripal A.V., Ponomarev D.V., Latysheva E.V., Nikitov S.A. // Proceedings of the 44th European Microwave Conf. 6-9 Oct 2014, Rome, Italy. P. 984-987.

[14] Joannopoulos I.D., Villenneuve Pierre R., Fan Shanhui // Nature. 1997. Vol. 386. N 13. P. 143-149.

[15] Усанов Д.А., Скрипаль А.В., Мерданов М.К., Горлищкий В.О. // ЖТФ. 2016. Т. 86. Вып. 2. С. 65-70. 